\title{
Epigastric Pain in an AIDS Patient; a Case of Misdiagnosis
}

\author{
Amira Mohamed ${ }^{1 *}$, Anita Lwanga ${ }^{1}$, Benjamin Levy ${ }^{2}$ \\ ${ }^{1}$ Resident Physician, Department of Internal Medicine, Mount Sinai Hospital, Chicago, IL 60608, USA \\ ${ }^{2}$ Gastroentrologist, Department of Internal Medicine, Mount Sinai Hospital, Chicago, IL 60608, USA
}

Received: May 2, 2016; Accepted: June 21, 2016; Published: July 30, 2016

*Corresponding author: Amira Mohamed, Department of Internal Medicine, Mount Sinai Hospital, 1500 S California Ave, Chicago, IL 60608,USA; Tel: 312-415-8809; E-mail: amira.mohamed@sinai.org

\begin{abstract}
Epigastric pain is one of the commonest complaints that primary care physicians and gastroenterologists encounter. With the lack of any alarm symptoms, the treatment is usually proton pump inhibitors which are mostly successful secondary to the prevalence of gastritis and gastroesophageal reflux disease. However, in certain scenarios, more aggressive investigations should be done.
\end{abstract}

We present a 61 year old female with AIDS who was in and out of hospitals for 4 months with epigastric pain and diarrhea. The patient had multiple endoscopies with no specific diagnosis made. During her last hospitalization, she underwent endoscopic retrograde cholangiopancreatography (ERCP) with sphincterotomy for suspected AIDS cholangiopathy which relieved her symptoms completely within 24 hours.

Because ERCP is considered an invasive procedure, it is rarely done without specific indications such as a suspected biliary stone or papillary stenosis however, a certain subgroup of patients may benefit from ERCP despite lack of specific signs. Sphincterotomy results in significant decrease in morbidity in AIDS cholangiopathy but should patients with AIDS undergo ERCP without definite indications? That is the question we raise after analyzing this stimulating case.

Keywords: AIDS cholangiopathy; Endoscopic retrograde cholangiopancreatography; ERCP; Double duct sign; Papillary stenosis

\section{Introduction}

AIDS cholangiopathy is an uncommon syndrome of acalculous cholangitis caused by opportunistic infections secondary to immunosuppression seen in untreated AIDS infections [1]. Since it is usually seen in patients with a CD4 count well below 100/ $\mathrm{mm} 3$, the incidence of AIDS cholangiopathy has decreased since the start of the era of effective anti retroviral therapy [2]. Biliary obstruction results from infection related strictures in the biliary tract causing right upper quadrant pain, epigastric pain and diarrhea; fever and jaundice are less common, seen in $20 \%$ of patients [3].

Characterized by multiple irregular narrowings, AIDS cholangiopathy is essentially indistinguishable from primary sclerosing cholangitis (PSC). There are four pathological patterns; combination of sclerosing cholangitis and papillary stenosis (50\%), isolated intrahepatic sclerosing cholangitislike appearance (20\%), isolated papillary stenosis (15\%), longsegment extrahepatic duct stricture +/- concurrent intrahepatic disease (15\%). Severe abdominal pain is indicative of papillary stenosis, while milder abdominal pain is usually associated with intrahepatic and extrahepatic sclerosing cholangitis without papillary stenosis [3].

The diagnosis is made by endoscopic retrograde cholangiopancreatography (ERCP). However, other studies (particularly ultrasound and magnetic resonance cholangiopancreatography [MRCP]) can be helpful in early evaluation and in selecting patients for ERCP [4]. Although infection is the most common cause of AIDS cholangiopathy, medical treatment directed against C. parvum, Microsporidium, or CMV, the commonest microbiological causes, does not influence symptoms or cholangiographic abnormalities. Thus, treatment of the cholangiographic abnormalities is primarily endoscopic, and the approach varies with the anatomic abnormality [5].

\section{Case Report}

We present a 61 year old female with HIV who has not taken antiretroviral therapy (ART) for over a year. The patient had been suffering from non specific epigastric pain for over 4 months which she described as burning pain 8/10 in severity, non radiating, intermittent, worse after eating and somewhat relieved by omeprazole which was prescribed by her gastroenterologist. She had 2 endoscopies in the past 9 months both of which were negative for any finding and she was told she had GERD however, for the past 2 weeks the patient developed non bloody non bilious diarrhea and vomiting so she presented to our hospital.

At the time of admission, the patient was cachexic and had diffuse abdominal tenderness otherwise no significant findings on exam. Her CD4 count was 7 with a viral load of 76861. She had normal liver enzymes (AST 27, ALT 8, ALP 61) however they increased in 2 days to AST 150, ALT 45 and ALP 279 with total bilirubin remaining normal throughout the admission.

A CT scan of the abdomen was ordered which showed a 'double duct sign'; the gallbladder was distended. There was both intrahepatic and extrahepatic biliary ductal dilation with 
the common bile duct measuring up to $1 \mathrm{~cm}$ in caliber. There is also dilation of the pancreatic duct with no definite obstructing mass or stone. Initially the patient was being treated for severe gastritis but with the elevation in liver enzymes and CT findings, an US of the gall bladder was ordered which showed mild dilation of the common bile duct $(8 \mathrm{~mm})$.

At that point, biliary disease was suspected and an ERCP was done for diagnosis and treatment, biopsies were obtained from the ampulla, duodenum, aspirate from the common bile duct and sent for acid fast stain, cytology and culture. The procedure revealed esophageal and gastric thrush but the papilla appeared normal. Nevertheless, given the dilation of the common bile duct and pancreatic duct, asphincterotomy was preformed which completely relieved the patient's symptoms.

A repeat CT of the abdomen was obtained prior to discharge which showed that the common bile duct was no longer dilated and the 'double duct' sign could no longer be visualized. Acid fast stain and cultures came back negative and cytology showed possible inflammation with focal atypia. The patient was enrolled in an HIV program and discharged with follow up with a primary care physician and a gastroenterologist.

\section{Discussion}

With the widespread use of ARTs, AIDS cholangiopathy is no longer the disease with poor prognosis and high morbidity. In fact, AIDS cholangiopathy is relatively uncommon [2] so clinicians would understandably consider more prevalent diagnoses first.

If AIDS cholangiopathy is suspected, ultrasound (US) is the most cost-effective initial study, with sensitivity for cholangitis ranging from 75 to 97 percent and specificity of up to 100 percent [4]. In one study, patients with suspected AIDS cholangiopathy and a negative US, ERCP was negative in over 90 percent of patients (17/18 patients) [4]. Based on such studies, ERCP are usually not performed on patients with normal liver enzymes and a negative US.

Nevertheless, another study concluded that only $75 \%$ of patients with AIDS cholangiopathy have abnormalities on an US $[2,6]$. From these studies it can be argued that all patients with high suspicion of AIDS cholangiopathy deserve an ERCP for both diagnosis and symptomatic treatment. It can also be suggested that such patients do not need any further testing prior to ERCP.

The only way to decrease mortality in AIDS cholangiopathy is controlling HIV using ART therapy [7]. Nonetheless, average survival is less than 3 years given severity of AIDS at this point [7].

Although ERCP does not cure the disease, nor does it decrease mortality, it does considerably decrease symptoms if a sphincterotomy is performed. Patients with high suspicion of AIDS cholangiopathy should have a low threshold for ERCP even if labs results and US suggest otherwise.

\section{Consent}

Informed consent was obtained from the patient.

\section{References}

1. Henry Knipe and A. Prof Frank Gaillard et al. AIDS cholangiopathy. Radiopaedia.org.

2. Bouche H, Housset C, Dumont JL, Carnot F, Menu Y, Aveline B, et al. AIDS-related cholangitis: diagnostic features and course in 15 patients. J Hepatol. 1993;17(1):34-39.

3. Cello JP and Chan MF. Long-term follow-up of endoscopic retrograde cholangiopancreatography sphincterotomy for patients with acquired immune deficiency syndrome papillary stenosis. Am J Med. 1995;99(6):600-603.

4. Daly CA and Padley SP. Sonographic prediction of a normal or abnormal ERCP in suspected AIDS related sclerosing cholangitis. ClinRadiol. 1996;51(9):618-621.

5. Hashmey R, Smith NH, Cron S, Graviss EA, Chappell CL, White AC Jr. Cryptosporidiosis in Houston, Texas. A report of 95 cases. Medicine (Baltimore). 1997;76(2):118-139.

6. Vakil NB, Schwartz SM, Buggy BP, Brummitt CF, Kherellah M, Letzer DM et al. Biliary cryptosporidiosis in HIV-infected people after the waterborne outbreak of cryptosporidiosis in Milwaukee. N Engl J Med. 1996;334(1):19-23.

7. Ducreux M, Buffet C, Lamy P, Beaugerie L, Fritsch J, Choury A, et al. Diagnosis and prognosis of AIDS-related cholangitis. AIDS. 1995;9(8):875-880. 